\title{
1979-2019: 40 años de democracia europea
}

\author{
1979-2019: 40 Years of European Democracy
}

\author{
Jaume Duch Guillot ${ }^{1}$ \\ Director-General de Comunicación y Portavoz del Parlamento Europeo \\ jaume.duch@europarl.europa.eu
}

Sumario: I. Introducción.-II. 1979: los ciudadanos europeos eligen por primera vez a sus representantes comunitarios._III. 1984: las elecciones de la Europa de los 10.-IV. 1989: nuevas normas y regulaciones para la Europa de los 12.-V. 1994: nuevos representantes europeos tras los acuerdos de Maastricht.-VI. 1999: la Europa de los 15 se prepara para los retos del siglo XXI.-VII. 2004: la Europa de los 25, unida en la diversidad del siglo XXI. - VIII. 2009: 1a Europa de los 27 elige un nuevo parlamento bajo el amparo del Tratado de Lisboa.-IX. 2014: una Europa de los 28 se enfrenta a desafíos cada vez más complejos.-X. 2019: la elección de la nueva Europa post-Brexit de los 27.-XI. Conclusiones.-XII. Bibliografía.

Resumen: Desde las primeras elecciones al Parlamento Europeo, en 1979, hasta las octavas, en 2014, la Unión Europea ha dado grandes pasos en su proceso de integración. En el presente artículo realizaremos un recorrido por la historia reciente de la democracia europea en el periodo comprendido entre estas fechas, desde las primeras elecciones europeas hasta las más recientes. En él incluiremos los resultados de cada convocatoria, la participación electoral en los diferentes Estados miembros y el contexto en el que se llevaron a cabo. Asimismo, explicaremos cómo los diferentes tratados comunitarios y las adhesiones de países miembros han ido modificando tanto la composición del hemiciclo, es decir, el número y distribución de eurodiputados en cada legislatura, como los poderes que la institución ha ido adquiriendo dentro de la Unión Europea. Finalmente, esbozaremos los retos y desafíos que deberá abordar la Unión Europea de cara a las próximas elecciones de mayo de 2019, 40 años después de los primeros comicios.

Palabras clave: Parlamento Europeo, elecciones europeas, Unión Europea, democracia

${ }^{1}$ Con la colaboración de Iria Pereira Paz y Ester Izquierdo Romagosa 
Abstract: Since the first elections to the European Parliament, in 1979, until the eighth ones, in 2014, the European Union has taken big steps in its integration process. In this article we will analyse the recent history of European democracy between these dates, from the first European elections to the most recent ones. We will analyse the results of each election, the turnout in the different Member States and the context in which they were held. Additionally, we will explain how the different treaties and the new Member States have modified both the composition of the hemicycle, that is, the number and distribution of MEPs in each term, as well as the powers that the institution has acquired within the European Union. Finally, we will outline the challenges that the European Union will have to face in the upcoming elections of May 2019, 40 years after the first ones.

Keywords: European Parliament, European elections, European Union, democracy

\section{Introducción}

La próxima primavera, concretamente entre el 23 y 26 de mayo de 2019 , tendrán lugar unos nuevos comicios europeos. Este mismo año se cumplen, además, 40 años desde las primeras elecciones al Parlamento Europeo, celebradas en 1979. Cuatro décadas de democracia europea que pocos años antes, con la II Guerra Mundial reciente, habrían parecido imposibles de lograr.

El proceso hasta conseguir que los ciudadanos europeos pudieran determinar directamente la composición de la mayor cámara supranacional del mundo no se dio de un día para otro. En primer lugar, haría falta que los países fundadores de la entonces Comunidad Económica Europea (la actual Unión Europea) se pusieran de acuerdo para conseguir una cooperación económica y social justa y que garantizara la paz entre ellos de forma definitiva. Una vez llegados a este consenso, dichos países deberían crear las diferentes instituciones de la Unión Europea que conocemos hoy en día. Entre ellas encontramos el Parlamento Europeo, que anteriormente tuvo el nombre de Asamblea Común (de 1952 a 1958) y Asamblea Parlamentaria Europea (de 1958 a 1962).

Durante esos primeros años, la cámara se componía de diputados designados por los parlamentos nacionales de los Estados miembros de entre sus propios miembros, no por los ciudadanos europeos. No fue hasta 1975, en la Cumbre de París, cuando los jefes de Estado y de Gobierno decidieron que se llevaran a cabo elecciones bajo sufragio universal si- 
multáneamente en todos los Estados miembros. Este acuerdo se validó jurídicamente en 1976 con la firma de la Decisión sobre el Sufragio Universal Directo para las Elecciones Europeas, el cual entró en vigor en 1978.

Las primeras elecciones al Parlamento Europeo tuvieron lugar en junio de 1979. Desde entonces, el proceso de elección ${ }^{2}$ de los distintos representantes europeos en la Eurocámara ha variado en múltiples aspectos, fundamentalmente en cuanto al número de ellos, en función de las sucesivas ampliaciones del número de Estados miembros. Si en la primera convocatoria electoral tan solo nueve estados componían la por entonces Comunidad Económica Europea, con un Parlamento formado por 410 diputados, en las últimas elecciones (2014) se eligieron 751 miembros representantes de los ciudadanos de 28 Estados miembros.

Los distintos procesos electorales también han experimentado diversas variaciones a raíz de la entrada en vigor de los sucesivos tratados comunitarios: Maastricht (1993), Niza (2003) y Lisboa (2009). Gracias a estas modificaciones de los Tratados institutores, el Parlamento Europeo ha ido adquiriendo cada vez más poderes dentro de la Unión Europea y logrado un mayor acercamiento entre la institución y la ciudadanía.

Para exponer el recorrido de estos últimos 40 años, el artículo se divide en nueve bloques, uno por cada convocatoria electoral europea. En cada apartado se recordarán los hechos más destacados de la anterior legislatura (en el caso de 1979, cómo se formó el Parlamento Europeo y se llegó a las primeras elecciones). También se harán constar los resultados finales y la composición del hemiciclo, así como la participación del electorado en los diferentes Estados miembros. En el caso de los comicios de 2019, se analizará la campaña de información que se está llevando a cabo y la nueva distribución de la Eurocámara en el caso de que finalmente se produzca la salida del Reino Unido de la Unión Europea.

\section{1979: los ciudadanos europeos eligen por vez primera a sus representantes comunitarios}

El proyecto común europeo presenta una larga trayectoria que se remonta al final de la II Guerra Mundial. Los horrores de la contienda, que desoló el viejo continente, derivaron en acuerdos entre antiguos rivales que buscaban restaurar la paz y consolidar la democracia.

2 «El Parlamento Europeo: modalidades de elección», Parlamento Europeo, última modificación en octubre de 2018, http://www.europarl.europa.eu/factsheets/es/sheet/21/el-parlamento-europeo-modalidades-de-eleccion. 
El primero de dichos acuerdos fue el Tratado del Benelux ${ }^{3}$, firmado el 5 de septiembre de 1944 por los gobiernos de Bélgica, Países Bajos y Luxemburgo, que en aquel entonces estaban exiliados en Londres a causa de la II Guerra Mundial. El pacto estableció una Unión Aduanera que no entraría en vigor hasta 1948. Gracias a esta alianza, el Benelux serviría de núcleo y empezaría a dar forma al llamado proceso de integración europea. Diez años más tarde, la cooperación económica entre los tres países se profundizaría aún más con el Tratado de Unión Económica del Benelux.

Como es bien sabido, la primera comunidad europea es la del Carbón y del Acero (CECA), que se constituye a través de la firma y ratificación del Tratado de París ${ }^{4}$ en 1951. Forman parte de la misma Alemania, Italia, Francia y los países del Benelux. La creación de la CECA, que entró en vigor el 23 de julio de 1952, también significó la puesta en marcha de las instituciones que, aun habiendo sido modificadas, son las mismas con las que contamos hoy en día: la Alta Autoridad (la actual Comisión Europea), el Comité Consultivo (actual Comité Económico y Social Europeo), el Consejo, el Tribunal de Justicia (actual Tribunal de Justicia de la Unión Europea) y la Asamblea Común (actual Parlamento Europeo).

La senda trazada por este primer tratado permitió en 1957 la ratificación del acuerdo que asentarían las bases de la actual Unión Europea: el Tratado de Roma ${ }^{5}$. Concretamente, el acuerdo estableció la Comunidad Económica Europea (CEE) y la Comunidad Europea de la Energía Atómica (Euratom), lo que se tradujo en la supresión de barreras comerciales y, paulatinamente, el derecho a la libre circulación de bienes, servicios, capitales y personas.

El tratado también acordó objetivos más específicos, siendo el primero y principal alcanzar una unión cada vez más estrecha entre los pueblos europeos; mejorar las condiciones de vida y laborales de los ciudadanos europeos; garantizar la competencia leal entre los mercados así como un intercambio comercial equilibrado; reducir las desigualdades sociales y económicas entre las diferentes regiones de la CEE, y respetar los fundamentos de la Carta de las Naciones Unidas ${ }^{6}$.

3 «Traité Benelux», Benelux, acceso el 10 de octubre de 2018», http://www.benelux.int/ fr/benelux-unie/nouveau-traite-benelux

4 «Tratado de París», Parlamento Europeo, acceso el 3 de septiembre de 2018, http:// www.europarl.europa.eu/about-parliament/es/in-the-past/the-parliament-and-the-treaties/treaty-of-paris

5 «Aniversario del Tratado de Roma: la Unión Europea cumple 60 años», Parlamento Europeo, última modificación el 24 de marzo de 2017, http://www.europarl.europa.eu/news/ es/headlines/eu-affairs/20170320STO67752/aniversario-del-tratado-de-roma-la-union-europea-cumple-60-anos

6 «Carta de las Naciones Unidas», Naciones Unidas, acceso el 5 de noviembre de 2018, http://www.un.org/es/charter-united-nations/index.html 
Con la creación de las dos comunidades (CEE y Euratom), la CECA amplió su Asamblea Común, la cual se constituyó el 19 de marzo de 1958, integrada por 142 miembros, bajo el nombre de Asamblea Parlamentaria Europea (no sería hasta 1962 cuando pasaría a denominarse Parlamento Europeo) y bajo la primera presidencia de Robert Schuman .

Sin embargo, aún tendrían que pasar 21 años desde la constitución de la Asamblea hasta que los ciudadanos pudieran hacer oír su voz, escogiendo directamente a sus representantes comunitarios.

Desde la entrada en vigor del Tratado de Roma, la por entonces Comunidad Económica Europea fue desarrollando paulatinamente su ámbito de competencias, a la vez que ampliando el número de Estados miembros. A los seis miembros fundadores (Bélgica, Países Bajos, Luxemburgo, República Federal de Alemania, Francia e Italia) se les unieron en 1973 tres nuevos estados: Dinamarca, Irlanda y Reino Unido. La Europa de los 9 iniciaba su andadura, pero aún faltaban seis años para que el Parlamento Europeo se convirtiera en una institución directamente elegida por los ciudadanos, tal y como la conocemos hoy en día.

El 9 y 10 de diciembre de 1974, los países miembros de la CEE se reunieron en la Cumbre de París ${ }^{7}$, donde decidieron celebrar tres reuniones anuales en calidad de Consejo Europeo, tal y como se recoge en la declaración del entonces presidente de la República francesa, Giscard d'Estaing: «Podemos decir que hemos asistido a la última Cumbre europea y que hemos participado en el primer Consejo Europeo» ${ }^{8}$.

También acordaron crear el Fondo Europeo de Desarrollo Regional y la Unión Económica y Monetaria ${ }^{9}$ y, lo más importante, que las elecciones al Parlamento Europeo, por sufragio universal y directo, deberían tener lugar a partir de 1978. Este acuerdo sería nuevamente validado, ya con valor jurídico, a través de la Decisión sobre el Sufragio Universal Directo para las Elecciones Europeas, suscrito en Bruselas el 20 de septiembre de 1976. Tras la ratificación de esta decisión por parte de los nueve Estados miembros, el acuerdo final entró definitivamente en vigor en julio de 1978.

7 «El Parlamento Europeo: evolución histórica», Parlamento Europeo, última modificación en octubre de 2018, http://www.europarl.europa.eu/factsheets/es/sheet/11/el-parlamentoeuropeo-evolucion-historica

8 «Déclaration du Président Giscard d'Estaing à la presse à l'issue de la réunion des chefs de gouvernement de la Communauté européenne à Paris (10 décembre 1974)», Centre Virtuel de la Connaissance sur l'Europe, última modificación el 5 de septiembre de 2012, https:// www.cvce.eu/content/publication/2005/2/21/6d60fe44-595d-481a-9e83-1ed2a5d9d790/publishable_fr.pdf

9 «La Historia de la Unión Europea - 1974», Unión Europea, último acceso el 6 de noviembre de 2018, https://europa.eu/european-union/about-eu/history/1970-1979/1974_es 
Es así como finalmente se llega a las primeras elecciones europeas, celebradas entre el 7 y el 10 de junio de 1979. Los 410 eurodiputados que componían por aquel entonces la Eurocámara fueron escogidos por sufragio universal directo, pero cada país siguió su propio sistema de voto, ya que no existía -y sigue sin existir más allá de algunos pocos elementos - una regla comunitaria común. La mayoría de los estados optó por una circunscripción única que representaba proporcionalmente el conjunto del país, pero otros miembros como el Reino Unido decidieron abordar las elecciones con diferentes circunscripciones.

La participación total en el conjunto de la Europa de los 9 fue de un $62 \%$. Esta cifra fue lógicamente superada ampliamente en países con voto obligatorio como Bélgica o Luxemburgo. En otros estados la participación fue más dispar. Por ejemplo, en Italia acudió a las urnas hasta un 85,7\% del electorado, mientras que en el Reino Unido apenas alcanzó el 32,4 \%.

Socialdemócratas, democristianos y conservadores fueron los partidos que coparon las tres primeras posiciones, con 112, 108 y 63 escaños, respectivamente. La sesión constitutiva de la Eurocámara dio lugar a lo que más tarde sería visto como un hito histórico: Simone Veil ${ }^{10}$ fue escogida primera presidenta del Parlamento Europeo elegido a través de sufragio universal directo. Fue asimismo la primera y única vez en que presidió la cámara una mujer, hasta que en 1999 fuera elegida la también francesa Nicole Fontaine.

\section{1984: las elecciones de la Europa de los 10}

La primera legislatura europea (1979-1984) permitió seguir avanzando en la construcción del proyecto común europeo, incluidas las políticas agrícola y comercial. Uno de los temas en los que se centró fue investigación y desarrollo, y muestra de ello es la puesta en marcha del programa comunitario ESPRIT, el primero de una larga lista de programas centrados en I+D. Este período supuso, además, la incorporación en 1981 de un nuevo socio comunitario: Grecia. Nacía así la Europa de los 10.

En este contexto de 10 estados se celebraron, entre el 14 y el 17 de junio de 1984, las segundas elecciones al Parlamento Europeo. Los comicios registraron una participación total del $59 \%$, tres puntos menos que en la anterior convocatoria electoral. Desde entonces y hasta 2009, la partici-

10 «Adiós a Simone Veil, primera mujer presidenta del Parlamento Europeo», Parlamento Europeo, última modificación el 30 de junio de 2017, http://www.europarl.europa.eu/ news/es/headlines/eu-affairs/20160811STO39006/adios-a-simone-veil-primera-mujer-presidenta-del-parlamento-europeo 
pación electoral iría descendiendo paulatinamente, hasta hacerlo abruptamente (cuatro puntos menos) con la primera participación de los Estados de Europa Central y del Este en 2004. Como veremos más adelante, el declive se frenó a duras penas en las elecciones de 2014, con una participación del $42,6 \%$, muy similar a la de los comicios precedentes.

En Grecia, el nuevo Estado miembro comunitario, acudieron a las urnas el 80,6\% de los electores, mientras que en Francia lo hicieron el 56,7 \% y en el Reino Unido, el 32,6\%. Sin normas generales comunitarias todavía bien definidas para los comicios europeos, el nuevo Europarlamento, formado por 434 eurodiputados, registró una nueva victoria electoral de los socialdemócratas europeos. Durante la legislatura, fue consolidándose el pacto entre socialdemócratas y democristianos sobre el que prácticamente se han basado hasta el día de hoy la mayor parte de los acuerdos en el seno de la cámara, incluido el reparto de la presidencia del Parlamento en dos períodos de dos años y medio cada uno.

\section{1989: nuevas normas y regulaciones para la Europa de los 12}

Pesca y agricultura, industria, transportes... La segunda legislatura europea (1984-1989) permitió la regulación a nivel comunitario de múltiples áreas. Una de ellas fue la educación, con la instauración del exitoso y referencial programa Erasmus en 1987, un proyecto que promueve tanto la formación de jóvenes europeos como la movilidad y cooperación entre los países miembros. En este periodo se produjo asimismo la incorporación de dos nuevos estados: España y Portugal, miembros de pleno derecho de la CEE desde el 1 de enero de 1986. Cabe reseñar que, entre el 1 de enero de 1986 y julio de 1987, ambos países estuvieron representados por eurodiputados nombrados por sus respectivas cámaras nacionales entre sus propios miembros; en el caso español, tanto diputados como senadores. A fin de no extender esa presencia más allá de lo razonable, los dos nuevos estados miembros celebraron elecciones europeas en junio de 1987, sin esperar el final de la legislatura.

1986 es también una importante fecha en la historia del proyecto común europeo. En febrero de dicho año, los 12 socios comunitarios firmaron el Acta Unica Europea ${ }^{11}$. Entre otros avances, el acuerdo sentaba las bases para un mercado único europeo, el fortalecimiento de una política común europea y la cohesión socioeconómica. Además, el Acta dotaba al Parla-

11 «'Acta Única Europea», Parlamento Europeo, acceso el 24 de septiembre de 2018, http://www .europarl.europa.eu/about-parliament/es/in-the-past/the-parliament-and-the-treaties/single-european-act 
mento Europeo de nuevas competencias, incluida la creación del llamado procedimiento de cooperación legislativa, que con los años daría lugar al procedimiento de codecisión que hoy en día conocemos. El nuevo procedimiento permitía a la Eurocámara participar en el proceso legislativo comunitario, a través de la presentación de enmiendas a los proyectos legislativos antes de su remisión al Consejo.

En este nuevo contexto tuvieron lugar las terceras elecciones europeas, celebradas entre el 15 y el 18 de junio de 1989. Un 58,4\% de los electores acudieron a votar, medio punto por debajo de los anteriores comicios.

En España y Portugal, los dos nuevos socios comunitarios (ambos con circunscripción electoral única que representa proporcionalmente al conjunto del país), la participación fue respectivamente del 54,7 \% y el 51,1\%. El Reino Unido volvió a alcanzar la cifra más baja: tan solo un 36,4 \% de sus electores acudieron a las urnas.

El Parlamento Europeo formado tras las elecciones de 1989 incluyó además un aumento considerable de escaños a causa de la incorporación de los dos países ibéricos: 518 eurodiputados componían la nueva cámara europea. De este conjunto total, los tres grupos políticos principales siguieron siendo los mismos de los años precedentes: 180 diputados se adscribieron al grupo de los socialdemócratas, 121 al de los democristianos y 49 al de los liberales.

\section{1994: nuevos representantes europeos tras los acuerdos de Maastricht}

La tercera legislatura europea (1989-1994) está ineludiblemente marcada por la caída del muro de Berlín el 9 de noviembre de 1989, que precipitó el final del comunismo en Europa Central y Oriental. Este acontecimiento derivó en la reunificación de Alemania, que a su vez supuso la posterior incorporación de la parte oriental de Alemania en la Unión Europea en 1990 y la desintegración del llamado Telón de Acero.

En este período destaca también la firma del histórico Tratado de Maastricht $^{12}$, suscrito por los 12 socios comunitarios el 7 de febrero de 1992. El también llamado Tratado de la Unión Europea supuso un paso de gigante para el proyecto común europeo, ya que transformó lo que era apenas una unión económica en una unión parcialmente política y social.

12 «Tratado de la Unión Europea (TUE) / Tratado de Maastricht», Parlamento Europeo, acceso el 25 de septiembre de 2018, http://www.europarl.europa.eu/about-parliament/es/inthe-past/the-parliament-and-the-treaties/maastricht-treaty 
Por un lado, el tratado permitió impulsar una política exterior y de seguridad común más ambiciosa que la llamada cooperación política europea, con el objetivo de proteger los valores comunes, los intereses fundamentales y la independencia de la UE; preservar la paz y la seguridad internacionales de acuerdo con los principios de las Naciones Unidas; promover la cooperación internacional, y desarrollar y consolidar la democracia y el Estado de Derecho, así como el respeto por los derechos humanos y las libertades fundamentales. También se fijaron objetivos en política interior, como el establecimiento de normas y controles para las fronteras exteriores de la UE; la lucha contra el terrorismo; la delincuencia organizada; el tráfico de drogas, y el fraude internacional.

Por otro lado, se empezó a hablar de una unión social gracias a la introducción del concepto de ciudadanía europea, lo que significaba, por ejemplo, que a cada ciudadano se le concedía el derecho a viajar y vivir libremente en cualquier lugar de la UE; votar y presentarse como candidato en las elecciones europeas y locales en su país de residencia; recibir asistencia y protección diplomática por parte de las embajadas y consulados de otros países miembros; formular peticiones al Parlamento Europeo, y enviar reclamaciones relativas a casos de mala administración de la UE al Defensor del Pueblo Europeo.

La incipiente unión económica se reforzó con la decisión de constituir una Unión Económica y Monetaria (UEM) alrededor de la moneda única europea, el euro. Esta decisión sentó las bases para que los países coordinaran sus políticas económicas, facilitaran una supervisión multilateral de esta coordinación y respetaran la disciplina financiera y presupuestaria. De este modo, la creación de la UEM permitió el libre flujo de capitales, abrió una cooperación en política económica y creó la moneda única, que llegaría a las manos de los ciudadanos el 1 de enero de 2002.

El Tratado de Maastricht, cuya entrada en vigor se produjo el 1 de noviembre de 1993, implicó también una nueva ampliación de competencias para el Parlamento Europeo. Gracias a este acuerdo, la Eurocámara adquiría el llamado poder de codecisión, es decir, una parte importante de la legislación europea solo podía aprobarse de común acuerdo entre el Parlamento y el Consejo. Se le otorgó asimismo un derecho de iniciativa legislativa indirecta, basado en el derecho de solicitar a la Comisión una propuesta legislativa sobre asuntos que considerara que requerían la adopción de un acto comunitario. En el ámbito interinstitucional, el Tratado reforzó enormemente al Parlamento en su relación con la Comisión Europea al otorgarle el poder de aprobar a todos los miembros de la Comisión, así como el de nombrar al Defensor del Pueblo Europeo para toda la legislatura.

Bajo este contexto tuvieron lugar, entre el 9 y el 12 de junio de 1994, las cuartas elecciones europeas. La nueva cámara europea, que pasaba a te- 
ner 567 escaños, volvería a estar copada mayoritariamente por socialdemócratas, democristianos y liberales: 214, 201 y 42 eurodiputados, respectivamente.

La participación total fue del $56,7 \%$, pero nuevamente se plasmó en datos muy dispares según el país: en España acudieron a los colegios electorales el 59,1\% de los votantes, mientras que en Portugal se alcanzó la cifra de participación más baja de la Europa de los 12: 35,5\%.

\section{1999: la Europa de los 15 se prepara para los retos del siglo XXI}

La cuarta legislatura europea (1994-1999) supuso la incorporación a la entonces recientemente denominada Unión Europea de tres nuevos países: Austria, Finlandia y Suecia. El ingreso de estos nuevos socios comunitarios, cuya adhesión se hizo efectiva en 1995, permitió alcanzar a las puertas del siglo XXI la llamada Europa de los 15.

La Unión Europea también logró un nuevo hito en este periodo con el Tratado de Ámsterdam ${ }^{13}$, firmado el 2 de octubre de 1997. Este acuerdo, en vigor desde el 1 de mayo de 1999, reforzaba el espacio de libertad, seguridad y justicia común, permitiendo por ejemplo la creación de una policía europea (Europol), fundada en 1998.

El acuerdo firmado en la capital holandesa otorgaba mayor notoriedad al Parlamento Europeo a través de un procedimiento de codecisión con el Consejo más simplificado y con un campo de aplicación más amplio, así como competencias de control y de cooperación reforzada. La Eurocámara también obtuvo el derecho de aprobación del presidente de la Comisión Europea ${ }^{14}$ que, con el subsiguiente desarrollo otorgado por el Tratado de Lisboa, ha supuesto un cambio de paradigma importante en las relaciones entre el Parlamento y la Comisión.

Bajo estas nuevas premisas, entre el 10 y el 13 de junio de 1999 se celebraron las quintas elecciones europeas. Por primera vez, la participación total $(49,5 \%)$ descendía por debajo del $50 \%$. Estos fueron también los primeros comicios europeos en los que, gracias a la ampliación del número de partidos nacionales integrantes, la primera fuerza política fue el Partido Popular Europeo y no la de los socialistas europeos. De los 626 eurodiputados

13 «Tratado de Ámsterdam», Parlamento Europeo, acceso el 28 de septiembre de 2018, http://www.europarl .europa.eu/about-parliament/es/in-the-past/the-parliament-and-the-treaties/treaty-of-amsterdam

14 «¿Cómo se nombra al presidente de la Comisión y a los comisarios?», Parlamento Europeo, acceso el 1 de octubre de 2018, http://www.europarl .europa.eu/news/es/faq/8/como-senombra-al-presidente-de-la-comision-y-a-los-comisarios 
electos, 233 correspondían a los cristianodemócratas y conservadores, 180 a los socialdemócratas y 50 a los liberales demócratas, los tres partidos mayoritarios de la Eurocámara de ese momento.

\section{2004: la Europa de los 25, unida en la diversidad del siglo XXI}

La Unión Europea ingresó en el siglo XXI en plena quinta legislatura (1999-2004). Fue en este periodo cuando se hizo efectiva la unión monetaria con la entrada en vigor del euro. A partir de 2002, esta nueva moneda se convirtió en la divisa oficial de 12 de los hasta entonces 15 miembros de la Unión Europea.

Dicho periodo está caracterizado también por el Tratado de Niza ${ }^{15}$, suscrito por la Unión Europea de los 15 en febrero del año 2001. El acuerdo, que entró en vigor de forma efectiva a comienzos de 2003, otorgó nuevamente más poderes legislativos y de control a la Eurocámara. El ejemplo más significativo fue la facultad que el Parlamento Europeo obtuvo de interponer recursos contra actos de la Comisión, el Consejo o el Banco Central Europeo, en casos de incompetencia, desviación de poder, violación del Tratado o de vicios sustanciales o de forma.

El acuerdo de Niza asentaba además los principios fundamentales para la ampliación de la Unión. De este modo, el 1 de mayo de 2004 se incorporaron 10 nuevos socios comunitarios: Estonia, Letonia, Lituania, Polonia, República Checa, Eslovaquia, Hungría, Eslovenia, Malta y Chipre. Nacía así la Europa de los 25.

El nuevo Parlamento Europeo, teniendo en cuenta todas estas adhesiones, pasaría a tener 732 escaños. La elección de los mismos se produjo entre el 10 y el 13 de junio de 2004, fecha de las sextas elecciones europeas. La participación total en estos comicios, un $45,5 \%$, fue de cuatro puntos inferior al anterior llamamiento a las urnas. Este descenso fue provocado fundamentalmente por la baja o bajísima participación en la mayor parte de nuevos Estados miembros.

Por países, nuevamente nos encontramos con resultados muy dispares: desde el $17 \%$ de participación en Eslovaquia al 71,7 \% de Italia o el 45,1 \% de España. Populares, socialistas y liberales-demócratas ocuparían, por este orden, los tres primeros puestos de la cámara de Estrasburgo, con 268, 200 y 88 eurodiputados, respectivamente.

15 «Tratado de Niza», Parlamento Europeo, acceso el 3 de octubre de 2018, http://www. europarl.europa.eu/about-parliament/es/in-the-past/the-parliament-and-the-treaties/treaty-ofnice 


\section{2009: la Europa de los 27 elige nuevo parlamento bajo el amparo del Tratado de Lisboa}

La sexta legislatura europea (2004-2009) trajo consigo nuevos cambios para la política europea. La Europa de los 25 pasó a ser de los 27 con la incorporación de Rumanía y Bulgaria el 1 de enero de 2007. Ese año también resultó clave con la firma, en el mes de diciembre, del Tratado de Lisboa ${ }^{16}$, que permitió avanzar en la construcción europea tras la no ratificación del Tratado por el que se establecía una Constitución para Europa $^{17}$. El tratado constitucional, a pesar de haber sido aprobado por la cámara europea en enero de 2005, había sido rechazado por Francia y los Países Bajos en mayo y junio del mismo año (respectivamente) en sendos referéndums nacionales, por lo que no pudo ser ratificado por los 27 países miembros y, por tanto, no había llegado a entrar en vigor.

El acuerdo suscrito en la capital portuguesa confería al Parlamento Europeo un rol legislativo más relevante, al reforzar y ampliar el ámbito de su poder de codecisión. La codecisión entre las tres instituciones, que se amplió a nuevos ámbitos, pasó a denominarse «procedimiento legislativo ordinario». Gracias al Tratado, los poderes legislativos de la cámara de Estrasburgo se equipararon con los del Consejo en diferentes campos, especialmente en la elaboración y aprobación de los presupuestos, la política agrícola y los asuntos de justicia e interior. Otra medida destacada fue la introducción de un sistema mediante el cual se permitía la participación de los Parlamentos nacionales en la activación o bloqueo de las iniciativas legislativas de la Comisión.

El Tratado de Lisboa no entraría en vigor hasta diciembre de 2009, seis meses después de las séptimas elecciones europeas. Dichos comicios, celebrados entre el 4 y el 7 de junio de 2009, tuvieron una participación total del $43 \%$ en el conjunto de la Unión Europea. El Parlamento Europeo resultante de dichas elecciones derivó en una Eurocámara formada por 736 escaños, de los cuales el Partido Popular Europeo, el Partido Socialista Europeo y el Partido Democrático Europeo volvieron a hacerse con los tres primeros puestos, con 265, 184 y 84 eurodiputados, respectivamente.

16 «Tratado de Lisboa», Parlamento Europeo, acceso el 28 de septiembre de 2018, http://www.europarl.europa.eu/about-parliament/es/in-the-past/the-parliament-and-thetreaties/treaty-of-lisbon

17 «Proyecto de Tratado por el que se instituye una Constitución para Europa (no ratificado)», Parlamento Europeo, acceso el 28 de septiembre de 2018, http://www.europarl.europa.eu/about-parliament/es/in-the-past/the-parliament-and-the-treaties/draft-treaty-establishing-a-constitution-for-europe 
Las elecciones de 2009 fueron además las primeras en las que la institución desarrolló una campaña de comunicación e incitación al voto coordinada en todos los estados miembros. Bajo el lema «It's your choice» («Tú eliges»), se desarrollaron diversas iniciativas para dar a conocer la importancia de las elecciones, se impulsó una mayor presencia en los medios de comunicación y se estrenaron asimismo las recién nacidas redes sociales a fin de llegar a un mayor número de ciudadanos.

\section{2014: una Europa de los 28 se enfrenta a desafíos cada vez más complejos}

En el transcurso de la séptima legislatura europea (2009-2014), surgieron en la Unión desafíos cada vez más complejos, entre los cuales destacan las consecuencias derivadas de la crisis financiera global. En este periodo se produjo además el ingreso de un nuevo país al club comunitario: Croacia, miembro de pleno derecho desde el 1 de julio de 2013.

La Europa de los 28 celebró las octavas elecciones europeas entre el 22 y el 25 de mayo de 2014. Además de la elección de 751 eurodiputados, la campaña electoral de 2014 contó con una importante novedad: cada partido político europeo podía presentar un candidato para presidir la Comisión Europea, lo que en la jerga comunitaria se conocía por el término alemán Spitzenkandidat. Se hacía así efectiva una de las novedades importantes del Tratado de Lisboa, que en su artículo $17.7^{18}$ estipula que el Parlamento Europeo elige al presidente de la Comisión Europea a propuesta del Consejo Europeo, «teniendo en cuenta los resultados de las elecciones europeas.» No todos estaban de acuerdo con que este precepto diese al Parlamento verdadera opción a la hora de decidir quién sería el presidente de la Comisión Europea, pero el consenso parlamentario a favor de una interpretación del tratado próxima al sistema de elección de muchos primeros ministros hizo que así fuera. A pesar de su incomodidad manifiesta, el Consejo Europeo se vio forzado a presentar como candidato a la presidencia de la Comisión a quien ya había sido bendecido como tal por las principales fuerzas políticas europeas al día siguiente de conocerse los resultados electorales.

La campaña de comunicación que se llevó a cabo para estos comicios tuvo como lema oficial «Act. React. Impact.» ${ }^{19}$ («Acción. Reacción. De-

18 Tratado de Lisboa por el que se modifica el Tratado de la Unión Europea y el Tratado Constitutivo de la Unión Europea (DOUE C 306 de 17 de diciembre de 2007)

19 «El poder para decidir el futuro de Europa», Parlamento Europeo, última modificación el 6 de septiembre de 2013, http://www.europarl.europa.eu/news/es/headlines/euaffairs/20130905STO18723/el-poder-para-decidir-el-futuro-de-europa 
cisión.»), si bien obtuvo más visibilidad el eslogan que se refería directamente a la elección del presidente de la Comisión, "Choose who's in charge $»^{20}$ («Tú puedes decidir quién dirige Europa»). El objetivo de ambos mensajes era hacer llegar a los ciudadanos la importancia, más que nunca en ese momento, de su participación en las urnas, pues el Parlamento Europeo había adquirido más competencias gracias al Tratado de Lisboa y, por tanto, los ciudadanos europeos también.

La campaña de 2014 contó, además, con varios debates, inéditos hasta la fecha, entre los diversos candidatos a la presidencia de la Comisión. El principal de todos ellos fue el llamado Eurovision Debate ${ }^{21}$, emitido por la Unión Europea de Radiodifusión (EBU, por sus siglas en inglés), en 49 televisiones europeas y en las 24 lenguas oficiales de la UE, el 15 de mayo de 2014. Participaron en el mismo los candidatos propuestos a presidir la Comisión Europea por parte de los distintos partidos europeos: Jean-Claude Juncker por el Partido Popular Europeo, Martin Schulz por el Partido Socialista Europeo, Guy Verhofstadt como candidato del Partido Liberal-Demócrata, Ska Keller por los Verdes y Alexis Tsipras por la Izquierda Unitaria Europea.

También se celebraron varios debates a dos entre otros candidatos, retransmitidos asimismo en directo por televisión, radio e internet.

En cuanto al sistema electoral empleado, a pesar de los distintos intentos de armonización impulsados por el propio Parlamento buscando mayor proporcionalidad, cada Estado miembro siguió su propio criterio. Países como España, Portugal o Grecia volvieron a optar por una única circunscripción proporcional a nivel nacional, mientras que otros socios comunitarios como Francia o Italia escogieron dividir su territorio en diferentes circunscripciones.

La participación total en los comicios de 2014 fue del 42,6\%, técnicamente la más baja registrada en toda la historia de las elecciones europeas, si bien en realidad el resultado era prácticamente el mismo obtenido cinco años antes, si la comparación se realizaba haciendo abstracción de la participación, por primera vez, de Croacia. Bruselas respiró, teniendo en cuenta que acababa de cerrarse la legislatura de la crisis económica y monetaria que tan duramente había afectado a muchos Estados miembros.

Nuevamente volvemos a encontrarnos con datos de participación muy diversos: desde el 74,8 \% de Malta hasta el 13,1\% de Eslovaquia, pasando

20 «Tú puedes decidir quién dirige Europa», Parlamento Europeo, última modificación el 16 de mayo de 2014, http://www.europarl.europa.eu/elections-2014/es/top-stories/choosewho-is-in-charge/

${ }^{21}$ «Eurovision Debate», Parlamento Europeo, última modificación el 16 de mayo de 2014, http://www.europarl .europa.eu/elections-2014/presidential-debate 
por el 43,8 \% de España o el 35,6\% del Reino Unido. Tanto 2009 como 2014 muestran asimismo una diferencia considerable entre el porcentaje de votantes en la antigua Europa de los 15 y la mayor parte de nuevos Estados miembros, por razones seguramente nacionales y que en otro momento podemos tratar con el suficiente rigor. De hecho, la participación electoral en los 15 Estados que ya eran miembros en 1999 gira desde esa fecha en torno al $47-49 \%$ de media, más de 15 puntos por encima de la media de los países de Europa Central y del Este.

Los resultados finales de estos comicios se saldaron nuevamente con una victoria del Partido Popular Europeo (221 escaños), seguidos del Partido Socialista Europeo (191) y, por primera vez, de los Conservadores y Reformistas Europeos (70), que desbancaron del tradicional tercer puesto a la familia liberal-demócrata ${ }^{22}$.

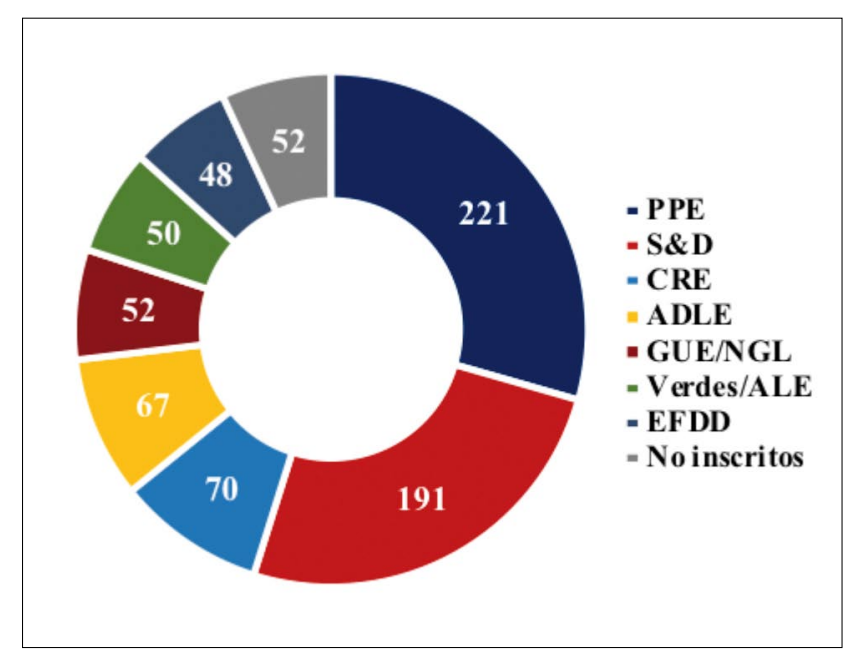

Resultados de las elecciones europeas de 2014 por número de eurodiputados

Fuente: elaboración propia a partir de la referencia citada.

22 «Resultados de las elecciones europeas de 2014», Parlamento Europeo, última modificación el 1 de julio de 2014, http://www.europarl.europa.eu/elections2014-results/es/electionresults-2014.html 


\section{2019: las elecciones de la nueva Europa post-Brexit de los 27}

La actual octava legislatura europea (2014-2019), que aún no ha concluido, introduce una modificación importante en lo que se refiere a la regulación de los procesos electorales a nivel comunitario. El 11 de noviembre de 2015, el Parlamento Europeo aprobaba la propuesta de reforma del Acta Electoral Europea, que posteriormente llegaría a la Mesa del Consejo. Como novedad, esta propuesta establece la obligatoriedad de incluir, tanto en las papeletas impresas como en los diferentes materiales de campaña, el nombre y el logotipo del partido al que está adscrita cada candidatura. Esta reforma también intentaba establecer medidas de coordinación de censos que hicieran posible, entre otras cosas, evitar que un mismo ciudadano pudiera votar varias veces en dos o más países diferentes.

A nivel social, la crisis de los refugiados, el auge de los populismos y el mayor alcance de las llamadas fake news son los principales asuntos que harán de estas elecciones unos comicios diferentes a todos los anteriores. La legislatura ha quedado marcada asimismo por un acontecimiento de especial relevancia para el futuro de la UE: la salida del Reino Unido. Mediante un referéndum celebrado el 23 de junio de 2016, el 51,89 \% de los británicos escogieron la opción que suponía la salida del Reino Unido de la Unión Europea.

En el momento de escribir este artículo el Reino Unido, a través de su Parlamento, todavía no ha decidido de qué manera abandonará la Unión Europea: con acuerdo, con una salida sin acuerdo u optando por una prórroga larga que supondría su participación en las elecciones europeas de mayo de 2019. La probable vuelta a la Europa de los 27 supone cambios relevantes en el Parlamento Europeo. La nueva Eurocámara pasará de 751 a 705 eurodipu$\operatorname{tados}^{23}$. De los 73 asientos que quedarán libres tras la salida del Reino Unido, 46 escaños se reservarán a futuras ampliaciones de la Unión Europea. Por su parte, los 27 escaños restantes se repartirán entre 14 países para mejorar la proporcionalidad y compensar los cambios demográficos de las últimas décadas: España recibirá cinco escaños más, al igual que Francia; Italia y los Países Bajos, tres más; Irlanda recibirá dos nuevos asientos adicionales, y Polonia, Rumanía, Suecia, Austria, Dinamarca, Eslovaquia, Finlandia, Croacia y Estonia recibirán, cada uno de ellos, un escaño más. ${ }^{24}$.

23 «El Parlamento Europeo reducirá su tamaño tras las elecciones de 2019», Parlamento Europeo, última modificación el 13 de junio de 2018, http://www.europarl.europa.eu/news/es/pressroom/20180607IPR05241/el-parlamento-europeo-reducira-su-tamano-tras-las-elecciones-de-2019

24 «Elecciones europeas: ¿cuántos eurodiputados tendrá cada país en 2019?», Parlamento Europeo, última modificación el 1 de febrero de 2018, http://www.europarl.europa.eu/news/ es/headlines/eu-affairs/20180126STO94114/elecciones-europeas-cuantos-eurodiputados-tendra-cada-pais-en-2019 
Número de eurodiputados según país y legislatura

\begin{tabular}{|c|c|c|c|}
\hline País & 2014-2019 & 2019-2024 & Variación \\
\hline Alemania & 96 & 96 & $=$ \\
\hline Austria & 18 & 19 & +1 \\
\hline Bélgica & 21 & 21 & $=$ \\
\hline Bulgaria & 17 & 17 & $=$ \\
\hline Chipre & 6 & 6 & $=$ \\
\hline Croacia & 11 & 12 & +1 \\
\hline Dinamarca & 13 & 14 & +1 \\
\hline Eslovaquia & 13 & 14 & +1 \\
\hline Eslovenia & 8 & 8 & $=$ \\
\hline España & 54 & 59 & +5 \\
\hline Estonia & 6 & 7 & +1 \\
\hline Finlandia & 13 & 14 & +1 \\
\hline Francia & 74 & 79 & +5 \\
\hline Grecia & 21 & 21 & $=$ \\
\hline Holanda & 26 & 29 & +3 \\
\hline Hungría & 21 & 21 & $=$ \\
\hline Irlanda & 11 & 13 & +2 \\
\hline Italia & 73 & 76 & +3 \\
\hline Letonia & 8 & 8 & $=$ \\
\hline Lituania & 11 & 11 & $=$ \\
\hline Luxemburgo & 6 & 6 & $=$ \\
\hline Malta & 6 & 6 & $=$ \\
\hline Polonia & 51 & 52 & +1 \\
\hline Portugal & 21 & 21 & $=$ \\
\hline República Checa & 21 & 21 & $=$ \\
\hline Reino Unido & 73 & 0 & -73 \\
\hline Rumanía & 32 & 33 & +1 \\
\hline Suecia & 20 & 21 & +1 \\
\hline Total & 751 & 705 & -46 \\
\hline
\end{tabular}

Fuente: elaboración propia a partir de la referencia citada. 
Este será el contexto en el que tendrán lugar las novenas elecciones europeas entre el 23 y el 26 de mayo de 2019.

La reducción del número de eurodiputados tras el Brexit tendrá repercusiones en algunos partidos: actualmente, 19 de 74 miembros de los Conservadores y Reformistas Europeos (CRE), y 19 de los 43 miembros de Libertad y Democracia Directo (EFDD) fueron elegidos en el Reino Unido en 2014.

El panorama político de las próximas elecciones se distinguirá del de las anteriores también por el auge de los populismos y extremismos que se ha producido en estos últimos años. Steve Bannon, ex-estratega de Trump, está preparando el lanzamiento del proyecto «The Movement», cuyo objetivo es formar un frente político común con los partidos de extrema derecha.

Las fuerzas euroescépticas o incluso eurófobas se han hecho un hueco en varios países de la UE y, por tanto, tienen influencia ya a través del Consejo de la Unión Europea. Sin embargo, habrá que ver cómo se organizan en el seno del Parlamento Europeo, pues hasta ahora han sido muy ineficaces y desorganizados.

Por otro lado, también están surgiendo nuevas fuerzas claramente proeuropeas, como es el caso del movimiento En Marche, liderado por el presidente francés, Emmanuel Macron. En el momento de escribir el artículo, todavía no está claro con qué grupo político se va a alinear, aunque se está empezando a hablar de una posible alianza con los liberales ${ }^{25}$.

Asimismo, en algunos países del continente se están formando listas para las elecciones europeas desde movimientos sociales que reivindican cambios en política y economía. El más destacado, también surgido en Francia, es el de los gilets jaunes (chalecos amarillos), que ha dado lugar a la creación de Ralliement d'initiative citoyenne (RIC).

Se constata una cierta tendencia a dejar de apoyar a las familias políticas tradicionales y decantarse por nuevas fuerzas. De hecho, populares y socialistas, los dos partidos europeos mayoritarios, representaban el $66 \%$ de los eurodiputados en la legislatura de 1999-2004, mientras que en esta última han supuesto el $54,8 \%$. Se prevé que este porcentaje baje aún más en la próxima legislatura pudiendo quedar incluso por debajo del $50 \%$.

Se espera pues una mayor fragmentación en el nuevo Parlamento, de modo que los diferentes grupos necesitarán recurrir a alianzas más amplias para conseguir la mayoría cualificada necesaria para adoptar legislación. No está claro en todo caso cuántos grupos políticos lograrán constituirse. Para

25 David M. Herszenhorn y Maïa de La Baume, «Macron and Rutte form liberal dream team», Politico, 10 de octubre de 2018, acceso el 30 de enero de 2019, https://www.politico. eu/article/emmanuel-macron-mark-rutte-liberal-dream-team-upend-european-politics 
formar un grupo político hace falta un mínimo de 25 eurodiputados de al menos un cuarto de los países miembros (actualmente 7). [Tal como especifica el artículo 32 del Reglamento interno del Parlamento Europeo].

Al igual que ocurrió en 2014, el Parlamento ha anunciado formalmente (informe González Pons, febrero de 2017) que elegirá al futuro presidente de la Comisión Europea de entre los candidatos oficiales que los diferentes partidos políticos europeos presenten antes de las elecciones, de manera que los ciudadanos puedan votar sabiendo qué partidos apoyan a qué candidatos.

El Presidente del Parlamento Europeo y la European Broadcast Union (EBU) firmaron en julio del pasado año un acuerdo ${ }^{26}$ que busca promover una mayor cobertura mediática de la campaña electoral europea de 2019. Gracias a este acuerdo se llevarán a cabo varias iniciativas, entre las que ya se ha confirmado una segunda edición del debate de candidatos a la presidencia de la Comisión. Una vez más, los Spitzenkandidaten expondrán sus prioridades y sus programas para el futuro de la UE en un debate que, al igual que en 2014, podrá seguirse en toda Europa vía televisión, medios en línea o redes sociales.

A diferencia de los anteriores comicios, en esta ocasión el Parlamento Europeo está llevando a cabo una campaña que no solo busca informar, sino que llama a la participación activa de los ciudadanos. Bajo el lema «This time I'm voting» ${ }^{27}$ («Esta vez voto») y disponible en los 24 idiomas oficiales de la UE, el Parlamento Europeo ha lanzado una plataforma cuyo objetivo es crear una comunidad de voluntarios que ayude a promover la participación en las próximas elecciones de forma consciente y plenamente informada. En otras palabras, no solo se pide a los europeos que voten, sino que animen a sus familiares, amigos y conocidos para que también lo hagan. De este modo, la campaña aprovecha el poder de la comunicación personal para hacer llegar su mensaje de forma más directa y efectiva.

Esta acción será complementada con otras más tradicionales, incluidas la emisión de un anuncio por radio y televisión, una fuerte presencia en los medios sociales y, sobre todo, vía la interacción con los medios de comunicación, el principal y más eficaz instrumento a la hora de dar a conocer a los ciudadanos europeos la importancia - esta vez ya indiscutible - de las próximas elecciones europeas.

${ }^{26}$ «EBU signs agreement with European Parliament on coverage of European Elections», European Broadcasting Union, última modificación el 11 de julio de 2018, https:// www .ebu.ch/news/2018/07/ebu-signs-agreement-with-european-parliament-on-coverage-ofeuropean-elections

27 «Esta vez voto», Parlamento Europeo, última modificación el 4 de septiembre de 2018, http://www .europarl.europa.eu/news/es/press-room/elections-press-kit/2/esta-vez-voto 


\section{Conclusiones}

En los cuarenta años transcurridos desde las primeras elecciones de 1979, el Parlamento Europeo ha ido aumentando en número de escaños como resultado de las diferentes adhesiones de los países a la UE; pero también, y más importante, ha ido reforzando sus competencias dentro de la arquitectura institucional de la Unión Europea. Las normas que regulaban el proceso de elección de los eurodiputados también han ido cambiando y evolucionando a lo largo de estos cuarenta años. En este largo periodo, los distintos cambios en los tratados han impulsado el proceso de construcción del proyecto europeo, al cual se han ido sumando nuevos países.

Las competencias de la Eurocámara han ido incrementándose, dando así más voz a los ciudadanos a través de la que es la única institución europea directamente elegida. Mientras que antes de 1979 eran los parlamentos nacionales de los Estados miembros los que escogían a los eurodiputados, desde esa fecha son cientos de millones de ciudadanos europeos los que deciden con su voto la composición del hemiciclo, e incluso tienen influencia en la elección del presidente de la Comisión Europea, gracias a la entrada en vigor del Tratado de Lisboa y a la interpretación que hace el Parlamento Europeo de su artículo 17.7.

Hace unas pocas décadas hubiese sido muy difícil imaginar un proceso electoral supranacional y con tan amplio alcance como es el de las elecciones al Parlamento Europeo, y sin embargo ahora se trata de un derecho sin el cual no es posible imaginar una Unión Europea democrática y que pueda contar con el respaldo de sus ciudadanos. Con el voto de cada europeo se decide el destino de Europa.

El Parlamento Europeo es el brazo democrático de la UE, y esta no puede dar la democracia por sentada. El camino que los europeos han ido construyendo durante cuarenta años es muy valioso, pero también reversible, como hemos visto en el caso del Brexit o en los comportamientos de algunos gobiernos poco respetuosos con los principios y valores que conforman la construcción europea. Se hace por ello más necesario que nunca poner en valor la importancia del impacto que ha tenido la construcción europea en la vida diaria de los ciudadanos y ser conscientes de que, con cada ciclo electoral, son los europeos los que deciden el futuro de este proyecto común.

Será la próxima primavera cuando los ciudadanos tendrán una oportunidad no solo de escoger a sus representantes en la Eurocámara sino también, a través de ese voto, de decidir hacia dónde quieren que vaya la Unión Europea y sobre qué vías deberá transitar. Ante los retos que presenta un mundo globalizado, a los que no tiene sentido enfrentarse en solitario, la ciudadanía europea deberá usar su voz y su voto para decidir su futuro colectivo. 


\section{Bibliografía}

Benelux. «Traité Benelux». Acceso el 10 de octubre de 2018». http://www.benelux. int/fr/benelux-unie/nouveau-traite-benelux

Centre Virtuel de la Connaissance sur l'Europe. «Déclaration du Président Giscard d'Estaing à la presse à l'issue de la réunion des chefs de gouvernement de la Communauté européenne à Paris (10 décembre 1974)». Última modificación el 5 de septiembre de 2012. https://www.cvce.eu/content/ publication/2005/2/21/6d60fe44-595d-481a-9e83-1ed2a5d9d790/publishable_ fr.pdf

European Broadcasting Union. «EBU signs agreement with European Parliament on coverage of European Elections». Última modificación el 11 de julio de 2018. https://www .ebu.ch/news/2018/07/ebu-signs-agreement-with-europeanparliament-on-coverage-of-european-elections

M. Herszenhorn, David, y de La Baume, Maïa, «Macron and Rutte form liberal dream team». Politico. 10 de octubre de 2018. Acceso el 30 de enero de 2019, https://www.politico.eu/article/emmanuel-macron-mark-rutte-liberal-dreamteam-upend-european-politics

Naciones Unidas. «Carta de las Naciones Unidas». Acceso el 5 de noviembre de 2018. http://www.un.org/es/charter-united-nations/index.html

Parlamento Europeo. «Adiós a Simone Veil, primera mujer presidenta del Parlamento Europeo». Última modificación el 30 de junio de 2017. http://www. europarl.europa.eu/news/es/headlines/eu-affairs/20160811STO39006/adios-asimone-veil-primera-mujer-presidenta-del-parlamento-europeo

Parlamento Europeo. «Aniversario del Tratado de Roma: la Unión Europea cumple 60 años». Última modificación el 24 de marzo de 2017. http://www.europarl. europa.eu/news/es/headlines/eu-affairs/20170320STO67752/aniversario-deltratado-de-roma-la-union-europea-cumple-60-anos

Parlamento Europeo. «¿Cómo se nombra al presidente de la Comisión y a los comisarios?». Acceso el 1 de octubre de 2018. http://www.europarl.europa. $\mathrm{eu} /$ news/es/faq/8/como-se-nombra-al-presidente-de-la-comision-y-a-loscomisarios

Parlamento Europeo. «Elecciones europeas: ¿cuántos eurodiputados tendrá cada país en 2019?». Última modificación el 1 de febrero de 2018. http:// www.europarl.europa.eu/news/es/headlines/eu-affairs/20180126STO94114/ elecciones-europeas-cuantos-eurodiputados-tendra-cada-pais-en-2019

Parlamento Europeo. «El Parlamento Europeo: evolución histórica». Última modificación en octubre de 2018. http://www.europarl.europa.eu/factsheets/es/ sheet/11/el-parlamento-europeo-evolucion-historica

Parlamento Europeo. «El Parlamento Europeo: modalidades de elección». Última modificación en octubre de 2018. http://www.europarl.europa.eu/factsheets/es/ sheet/21/el-parlamento-europeo-modalidades-de-eleccion

Parlamento Europeo. «El Parlamento Europeo reducirá su tamaño tras las elecciones de 2019». Última modificación el 13 de junio de 2018. http://www.europarl. europa.eu/news/es/press-room/20180607IPR05241/el-parlamento-europeoreducira-su-tamano-tras-las-elecciones-de-2019 
Parlamento Europeo. «El poder para decidir el futuro de Europa». Última modificación el 6 de septiembre de 2013. http://www.europarl.europa.eu/news/ es/headlines/eu-affairs/20130905STO18723/el-poder-para-decidir-el-futuro-deeuropa

Parlamento Europeo. «Esta vez voto». Última modificación el 4 de septiembre de 2018. http://www .europarl.europa.eu/news/es/press-room/elections-press-kit/2/ esta-vez-voto

Parlamento Europeo. «Eurovision Debate». Última modificación el 16 de mayo de 2014. http://www.europarl.europa.eu/elections-2014/presidential-debate

Parlamento Europeo. «L'Acta Única Europea». Acceso el 24 de septiembre de 2018. http://www .europarl europa.eu/about-parliament/es/in-the-past/theparliament-and-the-treaties/single-european-act

Parlamento Europeo. «Proyecto de Tratado por el que se instituye una Constitución para Europa (no ratificado)». Acceso el 28 de septiembre de 2018. http://www. europarl .europa.eu/about-parliament/es/in-the-past/the-parliament-and-thetreaties/draft-treaty-establishing-a-constitution-for-europe

Parlamento Europeo. «Resultados de las elecciones europeas de 2014». Última modificación el 1 de julio de 2014. http://www.europarl.europa.eu/ elections2014-results/es/election-results-2014.html

Parlamento Europeo. «Tratado de Ámsterdam». Acceso el 28 de septiembre de 2018. http://www .europarl.europa.eu/about-parliament/es/in-the-past/theparliament-and-the-treaties/treaty-of-amsterdam

Parlamento Europeo. «Tratado de la Unión Europea (TUE) / Tratado de Maastricht». Acceso el 25 de septiembre de 2018. http://www.europarl.europa. eu/about-parliament/es/in-the-past/the-parliament-and-the-treaties/maastrichttreaty

Parlamento Europeo. «Tratado de Lisboa». Acceso el 28 de septiembre de 2018. http://www .europarl .europa.eu/about-parliament/es/in-the-past/the-parliamentand-the-treaties/treaty-of-lisbon

Parlamento Europeo. «Tratado de Niza». Acceso el 3 de octubre de 2018. http:// www.europarl.europa.eu/about-parliament/es/in-the-past/the-parliament-andthe-treaties/treaty-of-nice

Parlamento Europeo. «Tratado de París». Acceso el 3 de septiembre de 2018. http:// www.europarl.europa.eu/about-parliament/es/in-the-past/the-parliament-andthe-treaties/treaty-of-paris

Parlamento Europeo. «Tú puedes decidir quién dirige Europa». Última modificación el 16 de mayo de 2014. http://www.europarl.europa.eu/elections-2014/es/topstories/choose-who-is-in-charge/

Tratado de Lisboa por el que se modifica el Tratado de la Unión Europea y el Tratado Constitutivo de la Unión Europea (DOUE C 306 de 17 de diciembre de 2007)

Unión Europea. «La Historia de la Unión Europea - 1974». Acceso el 6 de noviembre de 2018. https:/europa.eu/european-union/about-eu/history/19701979/1974_es 


\section{Sobre el autor}

Jaume Duch Guillot (Barcelona, 1962) es portavoz y Director General de Comunicación del Parlamento Europeo. Graduado en Derecho por la Universidad de Barcelona (1980-1985), donde después ejerció como profesor de Derecho Internacional Público. Es funcionario de la Unión Europea desde 1990, habiendo ejercido siempre tareas relacionadas con la información parlamentaria, entre ellas portavoz y consejero del Presidente Gil-Robles entre 1997 y 1999, responsable de información parlamentaria de las convenciones que redactaron la Carta de derechos fundamentales de la UE y el proyecto de Constitución Europea, jefe de la sala de prensa o Director de Medios (2006-2017). En 2017 fue nombrado Director General de Comunicación del Parlamento Europeo, un papel que combina con su rol como portavoz de la institución.

Jaume Duch habla español, francés, inglés, italiano y catalán y tiene nociones básicas de alemán. Es autor de diversas publicaciones, artículos y comunicaciones sobre cuestiones relativas a las instituciones de la Unión Europea y al derecho comunitario.

\section{About the Author}

Jaume Duch Guillot (Barcelona, 1962) is the Director-General for Communication of the European Parliament and its Spokesperson. He graduated in Law from the University of Barcelona (1980-1985), where he later also served as a Professor of International Public Law. In 1990 he became a European Parliament official and since then has always worked in communication-related positions, such as Spokesperson for the then President of the European Parliament, José María Gil-Robles, Head of the Press Room or Media Director, a position to which he was appointed in 2006. In February 2017 he was appointed as Director General of Communication, a role which he combines with being the Spokesperson of the institution.

Jaume Duch speaks Spanish, French, English, Italian and Catalan, has a basic knowledge of German. He is the author of various publications, articles and communications on issues related to the European Union, the role of the European Parliament and communication policies. 


\section{Derechos de autor}

Los derechos de autor (para la distribución, comunicación pública, reproducción e inclusión en bases de datos de indexación y repositorios institucionales) de esta publicación (Cuadernos Europeos de Deusto, CED) pertenecen a la editorial Universidad de Deusto. El acceso al contenido digital de cualquier número de Cuadernos Europeos de Deusto es gratuito inmediatamente después de su publicación. Los trabajos podrán leerse, descargarse, copiar y difundir en cualquier medio sin fines comerciales y según lo previsto por la ley; sin la previa autorización de la Editorial (Universidad de Deusto) o el autor. Así mismo, los trabajos editados en CED pueden ser publicados con posterioridad en otros medios o revistas, siempre que el autor indique con claridad y en la primera nota a pie de página que el trabajo se publicó por primera vez en CED, con indicación del número, año, páginas y DOI (si procede). Cualquier otro uso de su contenido en cualquier medio o formato, ahora conocido o desarrollado en el futuro, requiere el permiso previo por escrito del titular de los derechos de autor.

\section{Copyright}

Copyright (for distribution, public communication, reproduction and inclusion in indexation databases and institutional repositories) of this publication (Cuadernos Europeos de Deusto, CED) belongs to the publisher University of Deusto. Access to the digital content of any Issue of Cuadernos Europeos de Deusto is free upon its publication. The content can be read, downloaded, copied, and distributed freely in any medium only for non-commercial purposes and in accordance with any applicable copyright legislation, without prior permission from the copyright holder (University of Deusto) or the author. Thus, the content of CED can be subsequently published in other media or journals, as long as the author clearly indicates in the first footnote that the work was published in CED for the first time, indicating the Issue number, year, pages, and DOI (if applicable). Any other use of its content in any medium or format, now known or developed in the future, requires prior written permission of the copyright holder. 